\title{
Preferencias de formación continua entre los Titulados Universitarios en Ciencias de la Actividad Física y el Deporte en activo en Galicia
}

\author{
Lifelong learning preferences among University Graduates in \\ Physical Activity and Sport Sciences working in Galicia
}

\author{
Roberto Silva Piñeiro
}

Facultade de Ciencias da Educación e do Deporte, Universidade de Vigo (España).

\begin{abstract}
Resumen: La formación continua es una oportunidad de estar actualizado a los rápidos cambios en la adquisición de competencias que requiere el mercado laboral. Sin embargo, muchas veces la oferta no está acorde con las necesidades. Este artículo analiza los intereses formativos que tienen los profesionales Titulados Universitarios en Ciencias de la Actividad Física y el Deporte, y su relación con los ámbitos y salidas profesionales en el sector del deporte. Se utilizó una encuesta on-line en la que participaron 80 sujetos que actualmente ejercen en la Comunidad Autónoma de Galicia. El diseńo del cuestionario fu encargado un grupo de expertos. En Galicia los Titulados Universitarios en Ciencias de la Actividad Física y el Deporte en activo tienen mayor inclinación hacia la formación en los ámbitos de la Actividad Física y Salud, Entrenamiento, y Gestión Deportiva. Así mismo, prefieren formaciones específicas de duración intermedia y de carácter presencial.

Palabras clave: Formación continua, preferencias, actividad física, deporte.
\end{abstract}

Abstract: Lifelong learning is an opportunity to be updated to the rapid changes in the acquisition of skills required by the labor market. However, the supply is often not in line with the needs. This article analyzes the training interests of University Graduates in Physical Activity and Sport Sciences and their relationship with the professional fields and exits of the sports sector. An online survey was passed to 80 subjects who currently work in the Autonomous Community of Galicia. The design of the questionnaire was commissioned by a group of experts. In Galicia, University Graduates in Physical Activity and Sports Sciences are more inclined to training in the areas of Physical Activity and Health, Training, and Management of Sport. Likewise, they prefer specific formations of intermediate duration and face to face.

Keywords: Lifelong learning, preferences, physical activity, sport.

\section{Introducción}

La ubicación de la formación continua necesita referencias tanto a los estudios universitarios, como a los perfiles y ámbitos profesionales. Para todos aquellos que ya han finalizado la titulación universitaria y estén ejerciendo, la identificación de sus carencias formativas les permitirá anticipar las necesidades de actualización y mejora de competencias. La formación no es una obligación indiscriminada, sino una oportunidad de seguir creciendo en la profesión sin perder la situación del mercado laboral, permitiendo competir en mejores condiciones con más comodidad. Tanto desde el ámbito público como del privado será más productivo reconocer los perfiles de los trabajadores y poder ajustar sin demoras los programas formativos a sus demandas profesionales.

El antecedente del actual Grado Universitario en Ciencias de la Actividad Física y Deporte (en adelante CCAFD) es la Licenciatura en CCAFD, que a su vez proviene de la Licenciatura en Educación Física. La finalidad de la formación del Licenciado en CCAFD, elaborada por el Consejo Superior de Deportes (1991), era la especialización multidisciplinar en la motricidad humana, focalizándose en los aspectos forma-

Dirección para correspondencia [Correspondence address]: Roberto Silva Pińeiro. E-mail: roberto.silva@uvigo.es tivos, higiénicos, competitivos, recreativos y organizativos de la actividad física y el deporte. Se optó por una base común de conocimientos, acompańadas de asignaturas optativas a elección del estudiante, que ya desde los primeros planes de estudio disponían la posibilidad de especializarse, aunque en todas las universidades era posible, en función de unos itinerarios curriculares, semejantes a los ámbitos del mercado laboral: deporte, enseñanza, gestión y recreación, y salud (Rivadeneyra, 2000).

En los primeros planes de estudio de la Licenciatura de CCAFD a principios de los años 1990, no se hace todavía referencia a las competencias profesionales, ni al perfil profesional, ni a las capacidades profesionales. Los ámbitos de actuación profesional más utilizados eran docencia en educación física, entrenamiento deportivo, gestión deportiva, deporte extraescolar, investigación, actividad física y saludo, y recreación, y animación y turismo (Carratalá et al., 2004).

Cuando se solicitaba a los alumnos la incorporación de asignaturas en los itinerarios curriculares, ya mencionaban las nuevas tecnologías aplicadas al deporte, la alimentación y actividad física, marketing y publicidad, danza, procedimiento administrativo, o animación deportiva y deportes de aventura (Ribadeneyra, 1998). En este sentido, varios autores 
coincidían en introducir en el plan de estudios, formación en gestión deportiva (Blanco, 2003), actividad física para personas mayores (Cancela y Romo, 2001), nuevas tecnologías (Contreras y González, 2003), actividad física preventiva y de recuperación (García y Martín, 2002), motricidad acuática (Baixulí y Colado, 2001), y aquellas relacionadas con turismo deportivo, ocio, recreación, y actividades en el medio natural y costero (Latiesa, Paniza y Rebollo, 2002).

Las salidas al mercado laboral que disfrutaban una vez finalizada la Licenciatura en CCAFD entre los años 1999. 2001 (Carratalá, Mayorga, Mestre, Montesinos y Rubio, 2004), determinaba que el $46 \%$ trabajaban en docencia de la educación física; $15,6 \%$ en entrenamiento deportivo; $14,9 \%$ en gestión deportiva; el 9,5\% en actividad física y salud; y el $5,7 \%$ en recreación deportiva. El 51,9\% trabajaba en el sector público, el 41,8\% en el privado, y el 6,1\% eran autónomos. El $37,8 \%$ eran pluriempleados, por lo que requerían mayor desempeño profesional. A su vez, el 72,9\% realizaba cursos de perfeccionamiento después de acabar sus estudios, el 17,2\% másteres, el $16 \%$ otras carreras universitarias y el $26,9 \%$ estudios de doctorado.

El Tratado de Bolonia implicó una adecuación de las titulaciones al marco europeo, comenzándose a plantear que los cambios pudiesen atender a futuros profesionales. Hasta 2004 no se comenzarían a valorar en los planes de estudio del Grado de CCAFD las competencias que debería alcanzar cada uno de los egresados al final de su formación inicial para salir al mercado laboral. En ese momento, los itinerarios más realizados eran docencia de educación física, gestión deportiva, entrenamiento, y actividad física y salud; y los menos populares los de deporte y recreación, actividad física en el medio natural, iniciación deportiva, y expresión corporal (Campos, 2006).

Ante la tendencia a la especialización y adecuación de la formación a la demanda del mercado (Burriel, 1995; Mayorga, 1999; Contreras y González Ravé, 2003), han existido también partidarios de planes de estudios a favor de las competencias propias desde una perspectiva múltiple (técnica, pedagógica, cultural, y científica), a través de materias troncales y obligatorias en los primeros cursos, protegiéndose de especializaciones prematuras, que permitiesen una mejor formación en los ámbitos específicos de los cursos siguientes, y sobre todo, saber adaptarse a las situaciones y obligaciones profesionales en el futuro (Campos, 2006).
Poco a poco, el desequilibrio positivo en demanda y oferta de titulados universitarios en ciencias de la actividad física y el deporte que existía en los años 1990 se fue igualando, y fueron accediendo otros trabajadores no cualificados. Este fenómeno se ha agravado desde comienzo de siglo, pues el número de personas sin titulación específica o una insuficiente cualificación conllevó un desorden y saturación del mercado, en parte producido por la semejanza entre los perfiles y cualificaciones resultantes de las titulaciones que han ido surgiendo en estas décadas, la irrupción de un mercado de titulaciones no formales, la parcelación y control por parte de otras profesiones (médico deportivo, periodista deportivo...), y la ausencia de una normativa profesional en la actividad física y el deporte que determine los requisitos mínimos de cualificación y condiciones laborales. Sin embargo, a finales de los años 2000 seguían siendo varios los ámbitos profesionales en los que los egresados universitarios encontraban trabajo, pero no tenían una formación suficiente: docencia, dirección técnica, deporte, gestión, acondicionamiento físico, salud, ocio y recreación, investigación (Ibáñez, 2008).

El importante aumento de consumo deportivo y diversificación de la práctica deportiva conllevó la aparición de gran variedad de empleos relacionados con el sector deportivo, aunque la información que se dispone del mercado de trabajo no es lo suficientemente nítida como para poder obtener las explicaciones necesarias. Incluso si nos basamos en las estadísticas de las ocupaciones y profesiones relacionados con el deporte, que responden a aspectos económicos, no obtendremos una representación de todas las profesiones propias (Gambau, 2011).

Centrándonos en los egresados universitarios y de formación profesional, la demanda más fuerte se concentra durante la primera década una vez finalizados los estudios, aun teniendo en cuenta que los datos existentes no son suficientes, el perfil del demandante de empleo en el sector del deporte en Galicia en los últimos dos años responde a un joven de 26-35 años, excepcionalmente entre 36-55 años. Así también, los titulados universitarios que mayoritariamente demandan un trabajo lo hacen para ser gestor/director, monitor de yudo, preparador físico o profesor. Entre los directores y gerentes de centros deportivos, los mayores de 45 años son mayoría, sin embargo, entre los profesores de enseñanza secundaria son los menores de esa edad (Rodeiro, Gambau y Silva, 2017). 


\begin{tabular}{|c|c|c|c|c|}
\hline \multirow{2}{*}{ Profesión } & \multirow{2}{*}{ Nivel académico } & \multirow{2}{*}{ Contratos 2015} & \multicolumn{2}{|c|}{ Contrataciones } \\
\hline & & & $26-45$ años & $>45$ años \\
\hline Gestores y directores de centros deportivos & \multirow{5}{*}{$\begin{array}{c}\text { Titulado } \\
\text { Universitario }\end{array}$} & 85 & $5,6 \%$ & $26,1 \%$ \\
\hline Profesor Educación Física F. Profesional & & \multirow{2}{*}{1450} & $5,6 \%$ & $20,9 \%$ \\
\hline Profesores Educación Secundaria & & & $69,7 \%$ & $14,2 \%$ \\
\hline Preparadores físicos & & \multirow{6}{*}{3.060} & \multirow{6}{*}{$53,1 \%$} & \multirow{6}{*}{$17,9 \%$} \\
\hline Monitores de yudo & & & & \\
\hline Monitores de aparatos de gimnasio & \multirow{4}{*}{$\begin{array}{l}\text { Formación } \\
\text { Profesional }\end{array}$} & & & \\
\hline Entrenadores deportivos & & & & \\
\hline Monitores de mantenimiento físicos & & & & \\
\hline Monitores y animadores deportivos & & & & \\
\hline
\end{tabular}

Cuadro 1: Nivel académico y colectivos de contratación en el sector deportivo en Galicia (2014-2016).

Adaptado de Rodeiro, Gambau \& Silva (2017).

Entre 2014-2016 la tendencia de contratación en Galicia fue ascendente en un $12,2 \%$, lo que determina que hay menos personas en el sector esperando a encontrar un empleo, pero sobre todo en aquellas con un menor salario y mayor cantidad de contratos temporales como los instructores de actividades físico-deportivas y los monitores de actividades recreativas (Consejo COLEF, 2015; Observatori Catalá de l'Esport, 2014). Sin embargo, las ocupaciones de mayor pres- tigio y mejores condiciones, como son la gestión deportiva y la docencia, se encuentran saturadas.

A la espera de una normativa armonizadora nacional, las leyes autonómicas de regulación profesional del deporte establecen la referencia más actualizada de los perfiles profesionales que están manifestándose en el mercado laboral, y los niveles académicos a los que se accede.

\begin{tabular}{|c|c|c|c|c|c|}
\hline & \\
\hline & $\begin{array}{c}\text { Cataluña } \\
(2008 / 2015)\end{array}$ & $\begin{array}{l}\text { La Rioja } \\
\text { (2015) }\end{array}$ & Extremadura (2015) & $\begin{array}{c}\text { Andalucía } \\
(2015)\end{array}$ & Madrid (2016) \\
\hline Docente Educación Física & $\mathrm{L}$ & $\mathrm{L}$ & $\mathrm{L}$ & $\mathrm{L}$ & $\mathrm{L}$ \\
\hline Director/a deportivo/a & L-F-TS & L-TS & L-TS-O & L-TS & L-TS \\
\hline Gerente deportivo & & $\mathrm{L}-\mathrm{F}-\mathrm{O}$ & & & \\
\hline Preparador físico & & & $\mathrm{L}$ & & $\mathrm{L}$ \\
\hline Entrenador deportivo & L-TS-TD & L-TS-TD-O & TS-TD-O & L-TS-TD & TS-TD \\
\hline Monitor deportivo & L-E-F-M-O & & L-E-F-M-TS-TS-O & L-E-F-TS-O & L-F-M-TS-TD-O \\
\hline Educador físico & & $\mathrm{L}$ & & & \\
\hline Instructor deportivo & & L-E-F-TS-TD-O & & & \\
\hline Auxiliar de competición & & L-F-TS-TD-O & & & \\
\hline Técnico sin dedicación profesional & & L-F-TS-TD-O & & & \\
\hline Guía en el medio natural & & L-F-M-TS-TD-O & & & \\
\hline
\end{tabular}

Cuadro 2.: Resumen de perfiles profesionales en las leyes de regulación profesional del deporte en España.

L: TU CCAFDE; E: TU Educación Mención EF; F: TAFAD; M: Técnico Actividad Física Medio Natural; TS: Técnico Superior Deportivo; TD: Técnico Deportivo; O: Otros

Fuente: Consejo COLEF (2017).

Para organizar la formación continua es muy importante atender a su duración y metodología. A tal respecto, un modelo para discutir es el que se establece en la Ley Orgánica 2/2006, de 3 de mayo, de Educación, que creó una estructura para la formación inicial oficial de los técnicos deportivos. Esta normativa asume que la labor de estos técnicos requiere de actualización permanente, que alcance el nivel de exigen- cia en el rendimiento deportivo. Esta función se cedió a través del Consejo Superior de Deportes a las Federaciones Deportivas, que serán las encargadas de su formación continua. En particular, la primera propuesta fue el Programa de Formación Continua del Deporte (Programa FOCO) que comenzó el 1 de octubre de 2014 y finalizó el 31 de enero de 2017. Este programa considera los formatos orientativos de: Taller, con 
una duración mínima de 4 y máxima de 8 horas, distribuidas en 1 sola jornada; Seminario, con una duración de entre 8 y 20 horas y un mínimo de 2 jornadas; Curso monográfico, con una duración de entre 20 y 50 horas y un mínimo de 3 jornadas; y Congreso o simposio de carácter abierto.

\section{Metodología}

\section{Participantes}

La muestra estuvo compuesta por 80 Titulados Universitarios en CCAFD con una edad media de 34,2 años. Para este estudio se determinó que los participantes fuesen egresados de las Facultades de Ciencias de la Educación Física y del Deporte de la Comunidad Autónoma de Galicia, estuvieran en activo en uno o varios de los ámbitos de referencia (docencia, gestión deportiva, entrenamiento, recreación y actividad física para la salud), y dados de alta como ejercientes en el Colexio Oficial de Educadores Físico Deportivos de Galicia.

\section{Instrumento}

Se utilizó un formulario para la recogida de datos realizado específicamente para este trabajo, con la finalidad de hacer un análisis descriptivo.

Se desarrolló una primera fase para construir el instrumento, con base a los fines del estudio, redactando los ítems reactivos (Maica, Vañó, Pérez, y Ros, 2014; Blanco, 2016; Veiga, Valcarce y King, 2017; Bernabé, González \& Campos, 2018). En la segunda fase se sometió al juicio de 5 expertos, que procedían de población similar a quien se suministró el instrumento, y que valoraron el contenido y la forma de la herramienta. Los jueces constataron que la redacción era comprendida por el grupo objetivo, trasladando al investigador los aspectos a modificar (Soriano, 2015).

La primera versión del formulario estaba compuesto por 12 preguntas en 3 bloques, que sería reducido a 9 preguntas en dos bloques, después de la valoración de los expertos. Se redactaron 7 preguntas de opción mixta sobre temáticas formativas en un primer bloque, y 2 preguntas de opción cerrada multicotómica sobre la duración y modalidad de las formaciones en el segundo bloque.

\section{Procedimiento}

Se efectuó un contacto directo con varios profesionales cercanos para explicarles el propósito del estudio, que realizasen el cuestionario, y que nos trasladasen cualquier duda que pudiesen encontrar. A continuación, se realizó una llamada por correo electrónico a través del Colexio Oficial de Educadores Físico Deportivos de Galicia a todos aquellos profesionales que se encontraran en activo, comunicándoles que era un estudio totalmente anónimo y voluntario, e invitándoles a participar en el cuestionario on-line. El formulario estuvo abierto durante un período de 2 semanas, enviándose un recordatorio intermedio.

\section{Resultados}

Se presentan primeramente los resultados relacionados con las preferencias sobre ámbitos profesionales y temáticas, y seguidamente con la metodología y organización.

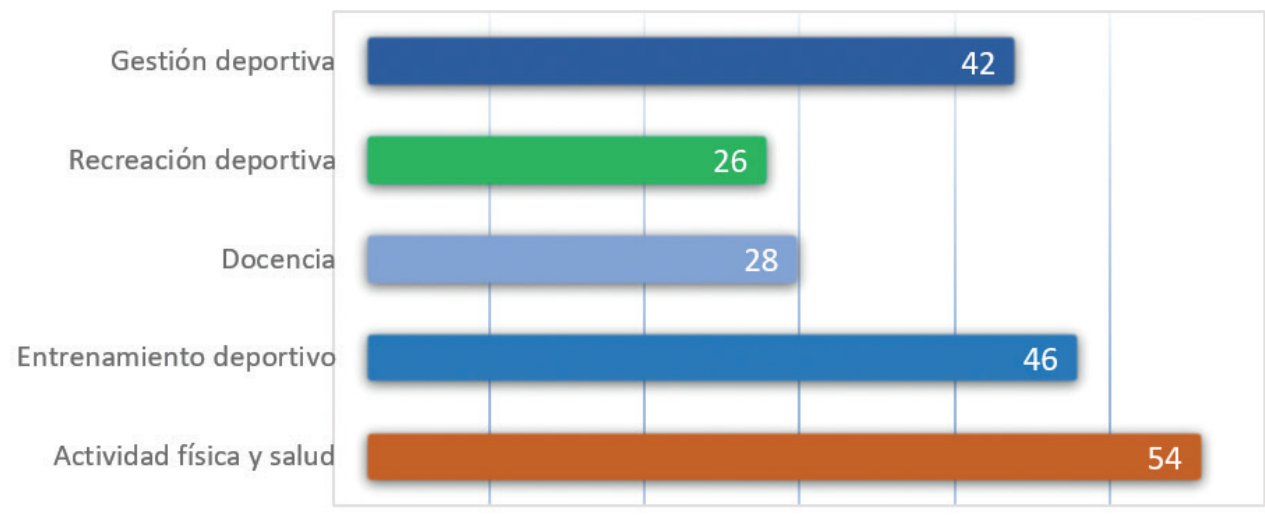

Gráfico 1: Ámbitos profesionales de mayor interés para recibir formación.

Según el gráfico 1, el 67,5\% (54) de los encuestados asegura que el ámbito de mayor interés es Actividad Física y Salud, el 57,5\% (46) el Entrenamiento deportivo y el 52,5\% (42) la
Gestión deportiva. Los menos valorados fueron la docencia y la recreación deportiva. 

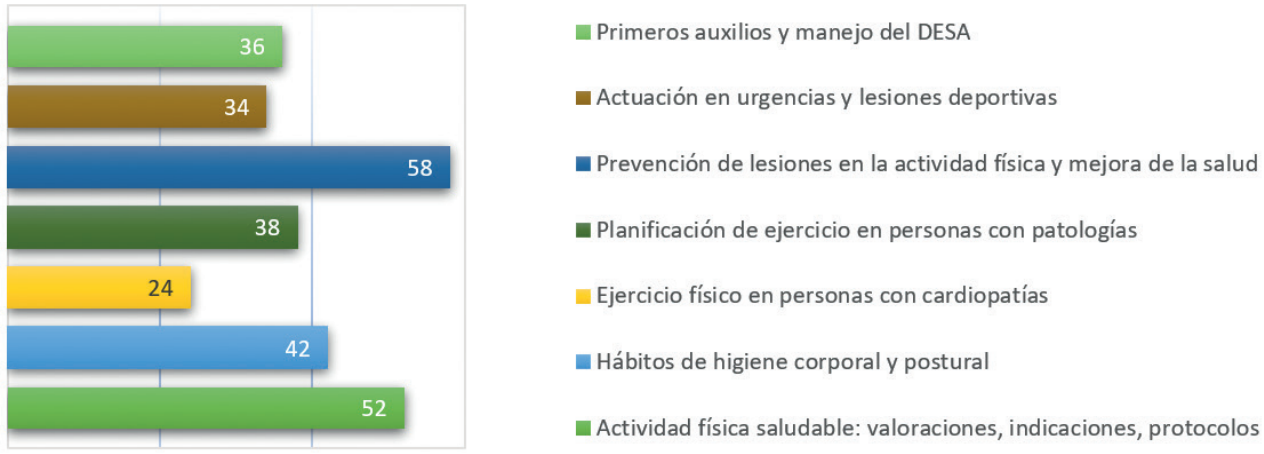

Gráfico 2: Preferencias sobre temáticas en Actividad Física y Salud.

Entre las temáticas sobre Actividad Física y Salud que se representan en el gráfico 2, destacan la "Prevención de lesiones en la actividad física y mejora de la salud" (72,5\%), la "Actividad física para la salud: valoraciones, indicaciones y protocolo" (65\%), los "Hábitos de higienes corporal y postural" (52,5\%), y también la "Planificación del ejercicio en personas con patologías" (47,5\%), puesto que varios encuestados consideran que también incluye al "Ejercicio físico para perso- nas con cardiopatías" (30\%). En particular, los participantes apuntan a la necesidad de experiencia práctica, y consideran importante la formación en fitness y actividades con soporte musical, pues es un ámbito en el que no se profundiza en las Facultades y en la formación no reglada está concentrada en Federaciones y entidades con estándares de calidad muy dispares.

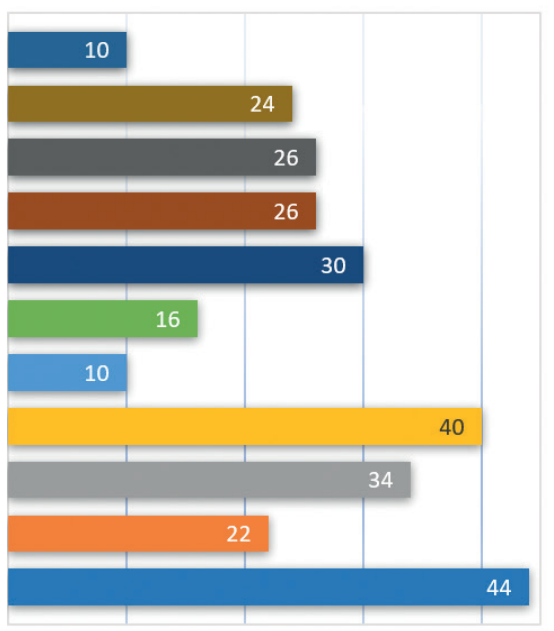

- Actividades acuáticas para población infantil

- Entrenamiento para población infantil

Ejercicio para personas mayores: antiaging

ש Electroestimulación aplicada al entrenamiento

- Actualización en metodologías de acondicionamento físico

- Acondionamiento físico en medio acuático

- Iniciación al entrenamiento en suspensión

- Programas de acondicionamiento físico personalizado

a Control del esfuerzo e intensidad en actividades físico-deportivas

Entrenamiento con pulsómetro

- Actualización en evaluación de la condición física

Gráfico 3: Preferencias sobre temáticas en Entrenamiento.

Los datos recogidos en el gráfico 3 muestran que las temáticas sobre entrenamiento más seleccionadas fueron la "Actualización en evaluación de la condición física" (55\%), "Programas de acondicionamiento físico personalizado" (50\%), y
"Control de esfuerzo e intensidad en actividad física" (42,5\%). Las formaciones relacionadas con el medio acuático, así como el entrenamiento en suspensión recibieron las peores valoraciones. 


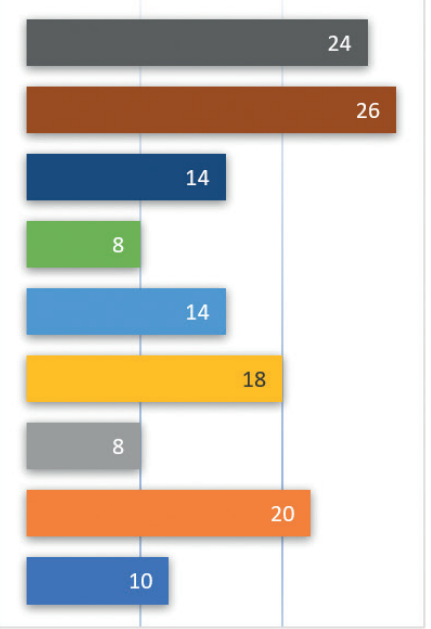

- Promoción de hábitos saudables en la educación física

- Actualización en metodologías de aprendizaje cooperativo en la EF

- El control emocional mediante el juego

- Juegos para la educación física: populares, alternativos, adaptados, deportivos

- Unidades didácticas de educación física en inglés

- Las TIC en educación física

Educación física inclusiva

- Como mejorar las habilidades del docente en educación física

- Actividades expresivas con base musical en educación física

Gráfico 4: Preferencias sobre temáticas en Docencia.

En el ámbito de la docencia, lo que más destaca es la preferencia por las "Metodologías de aprendizaje cooperativo en educación física" (32,5\%), y la "Promoción de hábitos saludables

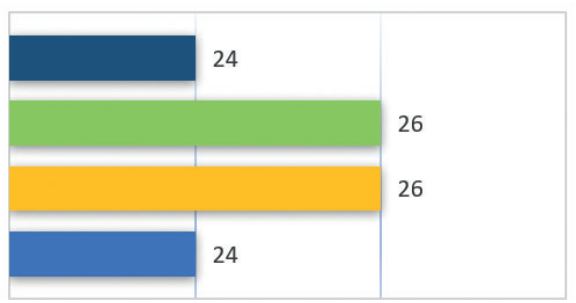

en educación física” (30\%). Sin embargo, temáticas como la "Educación física inclusiva" y los "Juegos para la educación física” apenas alcanzan al 10\% de los encuestados (Gráfico 4).

Gráfico 5: Preferencias sobre temáticas en Recreación.

En cuanto a las preferencias en el ámbito de la recreación, todas las temáticas presentan un interés para el 30-32,5\% de

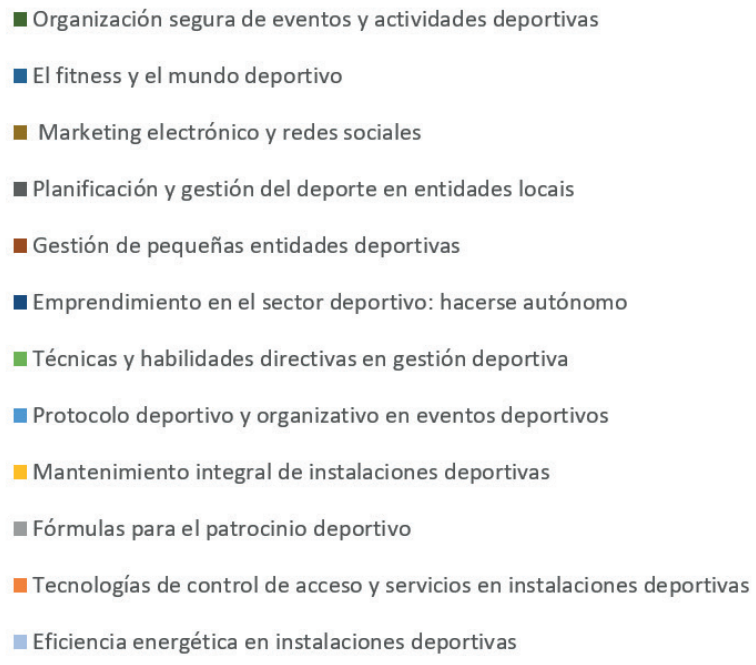

los encuestados. La muestra también indicó la opción de la iniciación a la escalada, dentro y fuera del aula (Gráfico 5).

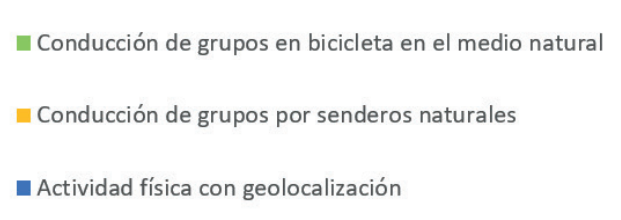

- Bienestar y rutas saludables por la natureza 
En cuanto a las preferencias temáticas relacionados con la gestión deportiva recogidas en el gráfico 6 , destaca la temática de "Protocolo deportivo" (40\%), "Fórmulas para el patrocinio deportivo" (37,5\%), "Técnicas y habilidades/emprendi- miento" (35\%), y "Fitness y mundo deportivo" (35\%). Menos interés suscitó la "Eficiencia energética en instalaciones deportivas" (10\%), o la "Planificación y gestión del deporte en entidades locales" (20\%).

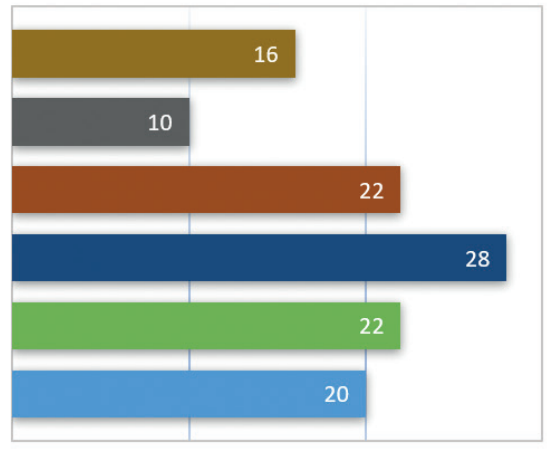

Deporte social y mujer

- Desigualdades en el ámbito de la actividad física y el deporte

- Metodologías de investigación en actividad física y deporte

- Nociones sobre alimentación y nutrición para la actividad física

- Protección de datos en entidades deportivas

n Correcta responsabilidad profesional

Gráfico 7: Preferencias sobre temáticas en Otras competencias.

Las temáticas de mayor interés para los encuestados sobre Otras competencias (Gráfico 7), fueron sobre "Nociones sobre alimentación y nutrición” (35\%), y las relacionadas con
"Metodologías de investigación" (27,5\%), "Protección de datos" (27,5\%), y "Responsabilidad profesional” (25\%).
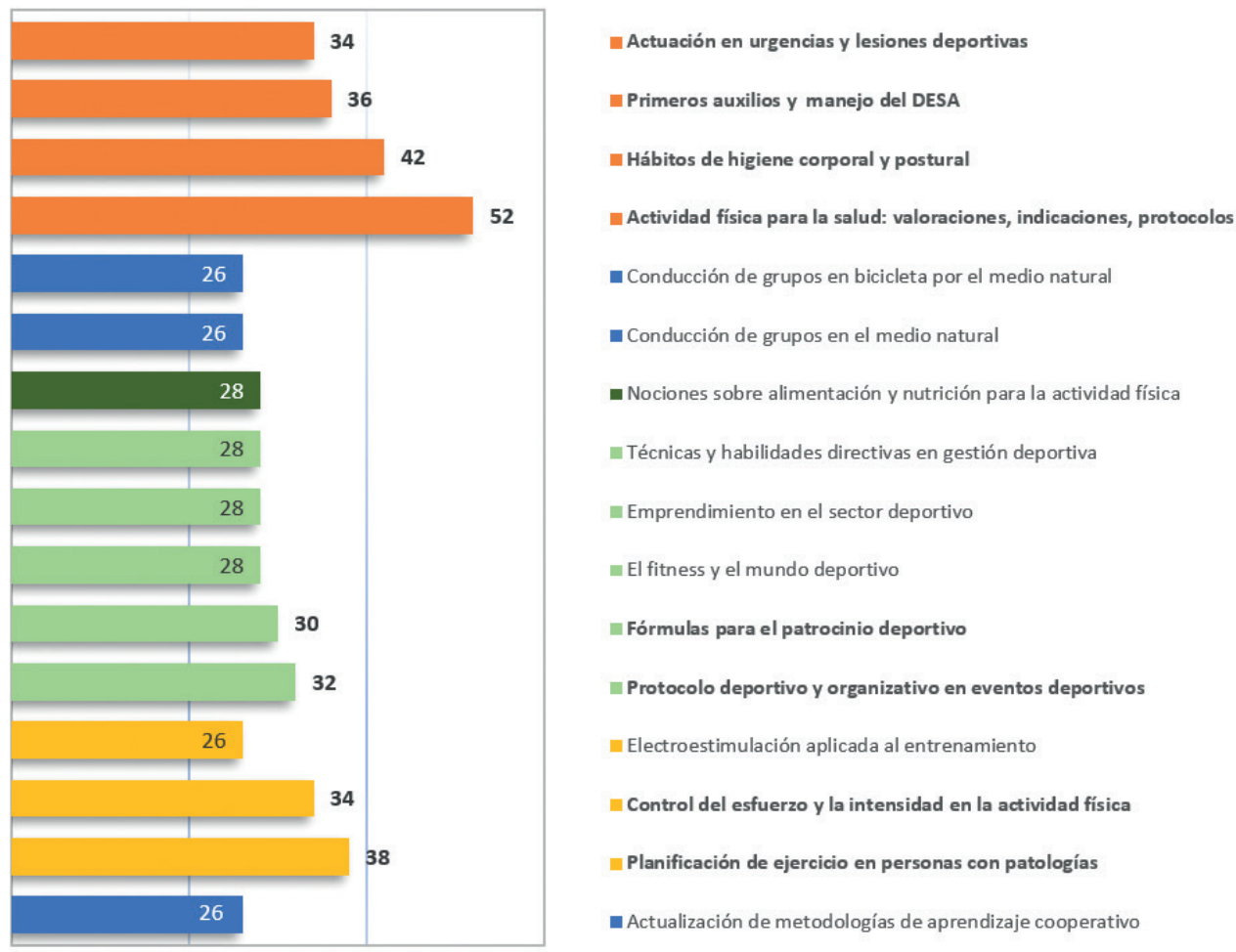

Gráfico 8: Resumen de las temáticas más seleccionadas.

Como se puede observar en el gráfico 8 , que recoge las temáticas que más interés indujeron, destacan las formaciones relacionadas con la Actividad Física y la Salud y el Entre- namiento. A continuación, las relacionadas con la Gestión deportiva, y finalmente aquellas referidas a la Recreación y la Docencia. 


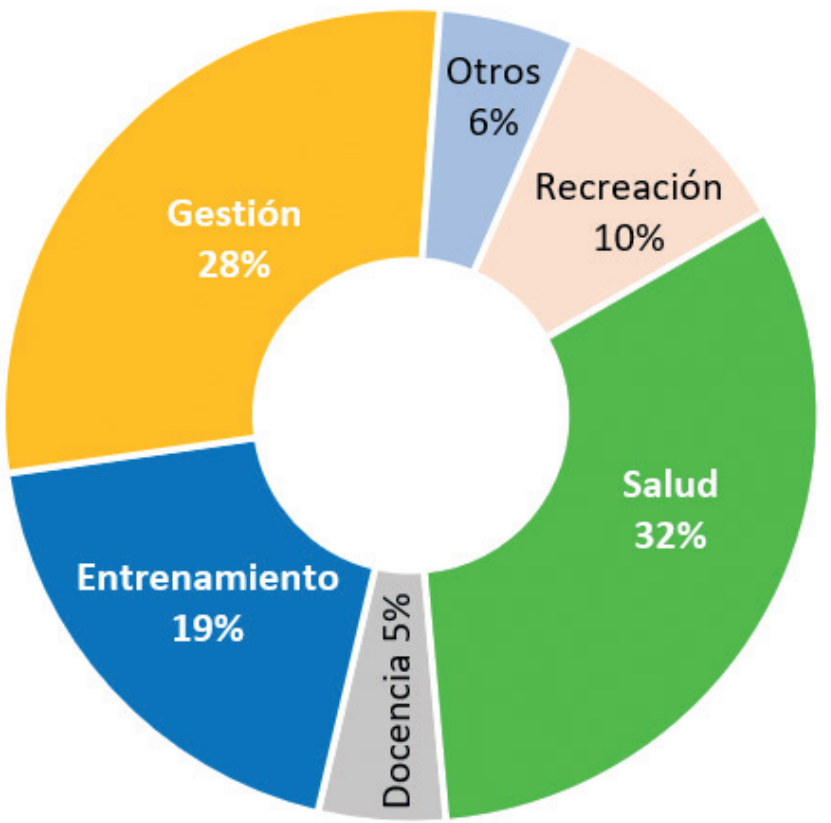

Gráfico 9: Comparativa de ámbitos más seleccionados en función de las temáticas formativas.

Para establecer la preferencia existente entre los ámbitos profesionales se realizó una comparación de las 4 primeras elecciones de cada uno (número mínimo alcanzado en todos), realizando el sumatorio total y ponderando los resultados parciales. Destaca una triada formada por el ámbito de Acti- vidad Física y Salud, seguida por el de Gestión Deportiva, y el de Entrenamiento. Con menos peso específico en este caso, se encuentran, la Recreación, la Docencia y Otras competencias (Gráfico 9).

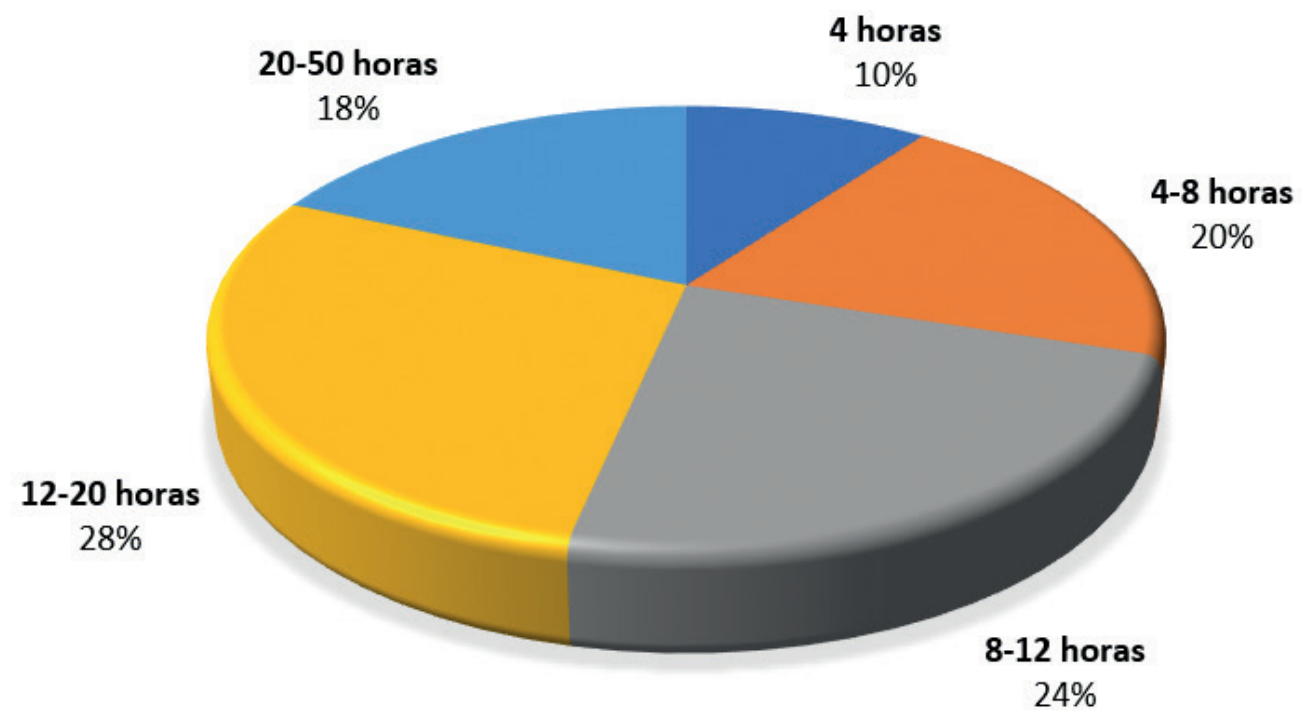

Gráfico 10: Duración de las acciones formativas.

Sobre la duración de las acciones formativas, el 72\% de los encuestados prefieren una duración entre 4 y 20 horas, y sobre todo el formato de 12-20 horas (28\%). Únicamente un
$10 \%$ se decantan por formaciones cortas hasta 4 horas, y un $18 \%$ por las largas entre 20 a 50 horas (Gráfico 10). 


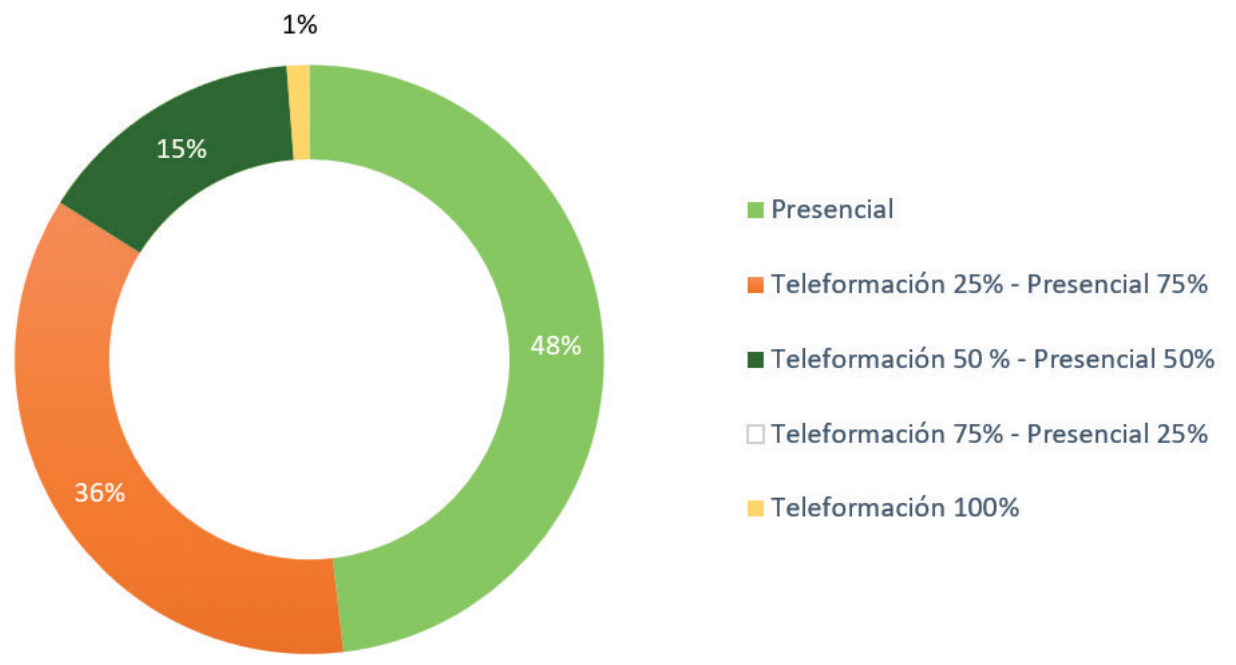

Gráfico 11: Metodología de aprendizaje.

Con relación a la metodología, el $48 \%$ de los encuestados se inclina por una formación presencial (48\%), y el 36\% por una presencial con un $25 \%$ a distancia (Gráfico 11 ).

\section{Discusión y conclusiones}

Las necesidades formativas de los Titulados Universitarios en CCAFD están marcadas fuertemente por las competencias adquiridas durante su formación, que depende de cada plan de estudios, y de las demandas del mercado laboral. Las primeras ocupaciones para los egresados más recientes suelen ser de instructores de actividades físico-deportivas y recreativas (Rocher, 2016), que irán iniciando la carrera profesional de cada persona, teniendo en cuenta que la movilidad laboral es cada vez mayor desde los primeros trabajos. Esto significa que desde su graduación universitaria el profesional tendrá que seguir formándose para cumplir con las exigencias laborales, máxime si su rotación en el puesto de trabajo es de menor duración. Es importante que los planes de estudio permitan alcanzar las competencias que faciliten una mayor capacidad de adaptación laboral, y que el propio egresado sea consciente de ir interpretando sus perspectivas e ir definiendo su propio camino formativo. Pero también es fundamental ser conscientes de la formación continua que necesitamos, pues su búsqueda y realización no será gratuita.

Para los estudiantes de la Licenciatura de CCAFD de la Universidad de Sevilla, las salidas profesionales preferidas son la gestión deportiva para el $33.1 \%$, el entrenamiento deportivo para el $21.2 \%$, y la actividad física y salud para el $20.3 \%$. Como segunda opción, el $36.4 \%$ eligió la docencia y el 15.3\% el entrenamiento deportivo (García, Pires y Fernández, 2013). Por su parte Gallo, González y Salinero (2010) comprobaron que tanto en la Universidad Camilo José Cela de Madrid (España) como en la Universidad de Hertfordshire (Inglaterra), la docencia y la gestión eran la primera opción de los estudiantes, mientras el entrenamiento la segunda. Asimismo, el 35.1\% de los alumnos de la Universidad Europea de Madrid optaban como primera opción por la docencia en educación física, seguido de la actividad física y salud con un $24.1 \%$ (Rodríguez et al., 2007). Algo similar sucedía en la Licenciatura de Ciencias del Deporte de la Universidad de Vigo, cuyos estudiantes se decantaban en primer lugar por la docencia y en segunda opción la actividad física y la salud (Cancela \& Ayán, 2010).

Según las preferencias de formación de este estudio, los tres ámbitos principales actualmente son la Actividad Física y Salud, Entrenamiento y Gestión deportiva. Las nuevas necesidades del mercado provocan una mayor diversidad de gustos profesionales, y se percibe que estas salidas anteriores se colocan en los primeros lugares entre las preferencias de los profesionales. Los ámbitos de la docencia y la recreación no tienen el mismo interés para la formación continua, lo que tiene relación, entre otras causas, con sus actuales expectativas laborales.

En Actividad Física y Salud y Entrenamiento, el empleo está viviendo un crecimiento importante, puesto que se perciben como pilares fundamentales para la calidad de vida, beneficiadas por la mayor preocupación por la salud y el fortalecimiento del deporte como pilar del desarrollo personal y social. Gracias a la promoción institucional y la mayor oferta deportiva y de instalaciones, personas de todas las edades tienen la oportunidad de acercarse de formas variadas a la práctica física, tanto por libre como supervisada. La sociedad percibe que existen profesionales preparados en estos ámbitos, que incluso colaboran con otras disciplinas, lo que genera mayor confianza y estabilidad en los servicios. Según García, Pires y Fernández (2013) ha crecido la demanda de estos servicios entre todos los sectores de la población (infantil, muje- 
res, mayores, personas con diversidad funcional, etc.).

El Titulado Universitario en CCAFD ha percibido que existen más oportunidades en estos ámbitos y que también necesita de mayor preparación, más allá de la recibida en la Facultad, pese a que en los planes de estudio se haya mejorado este aspecto.

La gestión ha sido un ámbito ocupado tradicionalmente por otros profesionales del mundo empresarial. En cuanto a la gestión deportiva, más reciente, se ha producido una incorporación importante de los Titulados Universitarios en CCAFD y han surgido perfiles propios, aunque no sólo se puede acceder desde la formación universitaria en Ciencias del Deporte, también desde otras formaciones superiores y medias. La evolución que ha sufrido este ámbito ha sido muy intensa, tanto desde la iniciativa pública como privada, lo que ha contribuido a multiplicar la promoción de la práctica física, conformar un sector productivo propio, y a visibilizar al resto de ámbitos profesionales para que la población pueda practicar con la seguridad y calidad que demanda.

En el caso de la docencia, por una parte, al proteccionismo que mantiene en los planes de estudio, la necesidad de realizar una especialización si se quiere acceder a la educación pública o concertada, y la caída y la dificultad de acceso a la oferta tanto pública como privada de empleo en el sector de la educación, lo que disminuye el atractivo que tenía en otros tiempos. En el estudio realizado con estudiantes por García,
Pires y Fernández (2013), también comprobaron que estaba disminuyendo el interés por la docencia, relacionándolo con los nuevos nichos laborales que les incitan a diversificar sus intereses profesionales.

Si nos referimos a la recreación, hablamos de un ámbito muy amplio, con menor presencia en los planes de estudio, con muchos competidores de otras profesiones, y con menor estabilidad y calidad laboral. A pesar de que tiene con gran potencial, no tiene la misma atracción que otros para los Titulados Universitarios en Ciencias de la Actividad Física y el Deporte, aparte de los problemas citados anteriormente, necesita una especialización intensa, y la figura profesional tampoco está suficientemente valorada.

En cuanto a la duración y la metodología de las formaciones, si nos referimos al Programa de Formación Continua del Deporte dirigido a técnicos deportivos y supervisado por el Consejo Superior de Deportes a través de las Federaciones Deportivas (MECD, 2014), únicamente considera válidas aquellas formaciones de corta duración (no debe superar las 50 horas ni las 8 jornadas de duración), y de metodología presencial.

En Galicia los Titulados Universitarios en CCAFD en activo tienen mayor inclinación hacia la formación en los ámbitos de la Actividad Física y Salud, Entrenamiento, y Gestión Deportiva. Así mismo, prefieren formaciones específicas de duración intermedia y de carácter presencial.

\section{Referencias}

1. Baixulí, A.M., \& Colado, J.C. (2001). Didáctica de la motricidad acuática en la formación inicial de los técnicos y profesores especialistas en Educación Física. En F.J. Canales, V. Mazón, D. Sarabia y F. Ruiz (Coords.) La enseñanza de la Educación Física y el deporte escolar. Actas del IV Congreso Internacional (pp. 365-370). Santander: A.D.E.F. Cantabria.

2. Bernabé, B., González, M., Campos, A. (2018): La formación continua formal de monitores españoles de actividad física y deporte. Apunts Educación Física y Deportes, 34, 134, 134-145.

3. Blanco, E. (2003). Nuevas tendencias en la gestión deportiva. Revista jurídica del deporte, 2(10), 355-362.

4. Blanco, E. (2016). Editorial: Tendencias en gestión deportiva. Revista Española de Educación Física y Deportes, 414, 13-16.

5. Burriel, J.C. (1995). Reflexiones sobre el futuro de los estudios de Actividad Física y Deporte. Ponencia presentada en las Jornadas de debate sobre el futuro de los estudios de Actividad Física y Deporte Barcelona, España. Cáceres: Universidad de Extremadura. Disponible en: http:// www.unex.es/ccdeporte/convergencia

6. Campos, A. (2006). Situación profesional de las personas que trabajan en funciones de actividad fisica y deporte en la Comunidad Autónoma Valenciana (2004). Universitat de València. Tesis Doctoral.

7. Cancela, J. M., \& Ayán, C. (2010). Profile and working expectations of Spanish physical education university students. Procedia Social and Behavioral Sciences, 2, 2487-2491.

8. Cancela, J.M., \& Romo, V. (2001). El envejecimiento de la población. Un condicionante en la formación de los Licenciados en Ciencias de la Actividad Física y del Deporte de Galicia. En R. Aranda, J.F. Campos Granell y S. Llana (Coords.), Nuevas aportaciones al estudio de la activi- dad física y el deporte, II (pp. 845-854). Valencia: Facultad de Ciencias de la Actividad Física y del Deporte de Valencia, Universidad de Valencia.

9. Carratalá, V., Mayorga, J., Mestre, J., Montesinos, J.M., \& Rubio, S (2004). Estudio del mercado laboral y de las competencias profesionales del titulado. En F. Del Villar (Coord.), Propuesta de Grado en Ciencias de la Actividad Física y el Deporte.

10. Consejo COLEF - Comisión Permanente y Equipo Profesional (2015) Memoria Anual 2015 Consejo COLEF. Versión 01. Disponible en: https://issuu.com/consejo-colef/docs/2015_-_memoria_consejo_colef

11. Consejo COLEF (2017). Leyes autonómicas de las profesiones del deporte. Disponible en: http://www.consejo-colef.es/ordenacion-profesional-home

12. Consejo Superior de Deportes (1991). Reforma de las enseñanzas y titulaciones deportivas. Propuesta para el debate. Madrid: CSD. Manuscrito no publicado.

13. Contreras, O.R., \& González Ravé, J.M. (2003). Evolució de les ten dències professionals del titulat en Educació Física i en Cièncias de l'Actividad Física i el l'Esport des del 1975 fins a l'actualitat. Apunts. Educació Física i esports, 73, 19-23.

14. Gallo, C., González, C., \& Salinero, J. J. (2010). Estudio comparativo de las motivaciones, percepciones y expectativas de futuro entre estudiantes de C.C.A.F.D. de la Universidad Camilo José Cela (España) y la Universidad de Hertfordshire (Inglaterra). Journal of Sport and Health Research, 2(3), 253-260.

15. Gambau, V. (2011). Deporte y empleo en Espańa: dificultades de estudio y de intervención. Revista Española de Educación Física y Deportes, 394(3), 13-36. 
16. García Merino, S., \& Martín Rodríguez, M. (2002). Vida activa y artrosis de rodilla: Papel del Licenciado en Ciencias de la Actividad Física y Del Deporte. Revista de Educación Física y Deportes, 9, 11-16.

17. García, J., Pires, F., \& Fernández, J. (2013): Preferencias profesionales de los estudiantes de licenciatura en Ciencias de la Actividad Física y del Deporte de la Universidad de Sevilla. Retos. Nuevas tendencias en Educación Física, Deporte y Recreación, 23, 39-42.

18. Instituto Nacional de Educación Física de Galicia (1994). Preámbulo al plan de estudios conducentes a la obtención del título oficial de Licenciado en Ciencias de la Actividad Física y Deporte. A Coruńa. Manuscrito no publicado.

19. Latiesa, M., Paniza, J.L., \& Rebollo, S. (2002). Deporte, turismo y salidas profesionales. En M. Latiesa y S. Rebollo (Eds.), Salidas profesionales del campo del turismo deportivo (pp. 15-32). Málaga: I.A.D. Málaga.

20. Luis Pascual, J.C. (2005). Las necesidades de formación del profesorado de educación física en educación primaria. Tesis Doctoral. Facultad de Educación. Universidad Complutense de Madrid.

21. Maica, L.E., Vañó, V., Pérez, J. \& Ros, C. (2014): Análisis de las tendencias formativas de los másteres en las facultades de CC de la Actividad Física y del Deporte en Espańa. FECIES 2013: X Foro Internacional sobre Evaluación de la Calidad de la Investigación y de la Educación Superior. Págs. 232-237.

22. Mayorga, J. I. (1999). Evolución de los planes de estudio de los licenciados en Ciencias de la Actividad Física y del Deporte. Tesis Doctoral. Departamento de didáctica, organización y didácticas escolares. Universidad Nacional Española a Distancia, España.

23. Ministerio de Educación, Cultura y Deporte (2014). Resolución de 19 de septiembre de 2014, de la Presidencia del Consejo Superior de Deportes, por la que se regula el Programa de Formación Continua del Deporte para el periodo 2015-2017 y se establecen las condiciones de incorporación de las Actividades de Formación Continua organizadas por las Federaciones Deportivas Espańolas. BOE núm. 237, de 30 de septiembre de 2014, páginas 77206 a 77213. Disponible en: https://
www.boe.es/boe/dias/2014/09/30/pdfs/BOE-A-2014-9885.pdf

24. Monroy, A. (2010). Los licenciados en administración de empresas y la gestión del deporte. Punto de Inflexión, 1, 1-8.

25. Monroy, A., \& Sáez, G. (2008). Las preferencias y expectativas de los estudiantes de actividad física y del deporte en la comunidad de Madrid. Cultura, Ciencia y Deporte, 3, 19.

26. Rivadeneyra, M.L. (1998). Perspectivas laborales y formación inicial de los Licenciados en Ciencias de la Actividad Física y Deporte de la Universidad de Granada. Tesis Doctoral, Facultad de Ciencias de la Actividad Física y el Deporte, Universidad de Granada, España.

27. Rivadeneyra, M.L. (2000). Análisis de la especialización de los Licenciados de la Actividad Física y Deporte en relación con las demandas del mercado laboral deportivo. En J.P. Fuentes \& M. Macías (Coords.) I Congreso de la asociación Española de Ciencias del deporte, II (pp. $513-$ 520). Cáceres: Universidad de Extremadura.

28. Rocher, M., Díaz, N., \& Tarragó, R. (2016). Análisis del Mercado laboral del deporte en Cataluña desde la perspectiva del cargo ocupado. Obsevatori Catalá de l'Esport - Institut Nacional d'Educació Física de Catalunya (INEFC).

29. Rodeiro, K., Gambau, V., \& Silva, R. (2017): Situación del empleo en el sector deportivo en Galicia (2014-2016). Revista Española de Educación Física y Deportes, 418, 49-64.

30. Rodríguez, G., Rivero, A., Montil, M., \& Garrido, M. (2007). Itinerarios profesionales de los estudiantes de ciencias de la actividad física y del deporte. Kronos, 12, 54-61.

31. Soriano Rodríguez, A. (2015). Diseño y validación de instrumentos de medición. Diá-Logos, 14, 19-40.

32. Veiga, O.L., Valcarce Torrente, M. \& King Clavero, A. (2017). Encuesta nacional de tendencias en fitness en España para 2017. Apunts. Educación Física y Deportes, 128(2),108-125.

33. Viñas, J., \& Vilanova, A. (2014). El mercat de treball en el context de l'esport a Catalunya. Especial incidència a la provincia de Barcelona. Barcelona: INDE. 